\title{
Nomogram for predicting advanced liver fibrosis and cirrhosis in patients with chronic liver disease
}

\author{
Rongrong Ding ${ }^{1}$, Xinlan Zhou ${ }^{1}$, Dan Huang ${ }^{1}$, Yanbing Wang ${ }^{1}$, Xiufen Li ${ }^{1}$, Li Yan ${ }^{1}$, Wei Lu' ${ }^{1}$ Zongguo Yang ${ }^{2^{*}}$ (D) and \\ Zhanqing Zhang ${ }^{1 *}$ (i)
}

\begin{abstract}
Background: We aimed to formulate a novel predictive nomogram to discriminate liver fibrosis stage in patients with chronic liver disease.

Methods: Nomograms were established based on the results of multivariate analysis. The predictive accuracy of the nomograms was assessed by ROC analysis and calibration. Decision curve analysis (DCA) was used to determine the clinical benefit of the nomograms.

Results: INR, platelets, and N-terminal propeptide type III collagen (PIIINP) were independent predictors for advanced liver fibrosis ( $\geq$ S3) and cirrhosis (S4) in patients with chronic liver disease in the training cohort. In the training set, the areas under the ROCS (AUROCS) of nomogram S3S4, APRI, FIB-4, and GPR for stage $\geq$ S3 were $0.83,0.71,0.68$, and 0.74 , respectively; the AUROCs of nomogram S4, APRI, FIB-4, and GPR for stage $S 4$ were $0.88,0.74,0.78$, and 0.79 , respectively. The calibrations showed optimal agreement between the prediction by the established nomograms and actual observation. In the validation set, the AUROCs of nomogram S3S4, APRI, FIB-4, and GPR for stage $\geq$ S3 were 0.86, 0.79, 0.78 , and 0.81 , respectively; the AUROCs of nomogram S4, APRI, FIB-4, and GPR for stage S4 were $0.88,0.77,0.81$, and 0.83 , respectively. Furthermore, the decision curve analysis suggested that the nomograms represent better clinical benefits in both independent cohorts than APRI, FIB-4, and GPR.
\end{abstract}

Conclusion: The constructed nomograms could be a superior tool for discriminating advanced fibrosis and cirrhosis in chronic liver disease.

Keywords: Nomogram, INR, Platelet, Liver fibrosis, Chronic liver disease

\section{Background}

Chronic liver disease is a major public health problem that accounts for high morbidity and mortality worldwide. Their prognosis and treatment depend largely on the progression of liver fibrosis. The timely and accurate assessment of liver fibrosis is essential to prevent chronic

\footnotetext{
*Correspondence: yangzongguo@shphc.org.cn; doctorzzqsphc@163.com ${ }^{1}$ Department of Hepatobiliary Medicine, Shanghai Public Health Clinical Center, Fudan University, 2901 Caolang Road, Shanghai 201508, China

2 Department of Integrative Medicine, Shanghai Public Health Clinical Center, Fudan University, 2901 Caolang Road, Shanghai 201508, China
}

liver disease from developing into cirrhosis, hepatic failure, and hepatocellular carcinoma [1]. To date, liver biopsy is still considered the gold standard for the assessment of liver fibrosis. Nevertheless, liver biopsy is an invasive procedure with some limitations, such as potentially life-threatening complications, high cost, observer discrepancies, and sampling errors [1]. Radiological methods including conventional ultrasound, computed tomography and magnetic resonance imaging can diagnose typical liver cirrhosis but cannot evaluate the presence or degree of liver fibrosis. 
Therefore, many efforts to develop noninvasive methodologies that reflect the total amount of liver fibrosis have been made. Blood markers such as platelets and hyaluronic acid have been reported to be related to liver fibrosis [2, 3]. Serological markers such as the aspartate transaminase to platelet ratio (APRI), fibrosis-4 score (FIB-4), and $\gamma$-glutamyl transpeptidase to platelet ratio (GPR) have been used to assess liver fibrosis and have been reported to be effective [4-6]. However, they are controversial with respect to the evaluation of liver fibrosis stages [7-9]. Transient elastography has been developed as a procedure able to assess hepatic stiffness noninvasively $[10,11]$. However, some factors, such as the need for expensive equipment and trained operators, may limit its clinical application [12]. Additionally, other potential mingle factors may also affect the accuracy of $\mathrm{TE}$, such as obesity, increased transaminase, and operator skills [13].

A nomogram is an effective model for visualizing regression equations because it can establish scoring standards based on the regression coefficients of all independent variables [14]. Compared with other prediction models, prediction based on nomograms is more accurate. This method has been proposed as an alternative method or even a new standard method and has been widely used as a prognostic tool for many tumor types, such as hepatocellular carcinoma, lung cancer, renal cell carcinoma, and colorectal cancer [15-19].

The aim of our study was to explore the predictive significance of serological indexes such as serum fibrosis markers, coagulation factors, aminotransferase, bilirubin, and platelets for staging liver fibrosis. Furthermore, we wished to establish a predictive nomogram based on fibrosis indicators with prognostic significance. In addition, a comparison was made between the nomogram and other noninvasive models, including ARPI, FIB-4, and GPR.

\section{Methods \\ Patients}

Overall, between January 2016 and September 2020, we retrospectively recruited 701 consecutive Chinese individuals with chronic liver disease. The enrolled patients were divided into two time groups; the first 427 patients admitted to our hospital between January 2016 and December 2019 formed the training set, while the remaining 274 patients admitted between January 2020 and September 2020 formed the validation set. All patients underwent a careful clinical examination and liver biopsy at Shanghai Public Health Clinical Center, Fudan University. The inclusion criteria were diagnosed patients with treatment-naïve chronic viral hepatitis (hepatitis B or C), alcoholic liver disease, nonalcoholic fatty liver, and autoimmune hepatitis. All the patients were $>18$ years old. The exclusion criteria were hepatocellular carcinoma, antiviral treatment history, decompensated cirrhosis, inadequate liver biopsy samples $(<1.5 \mathrm{~cm})$, and the use of anticoagulant drugs.

\section{Liver biopsy}

Percutaneous liver biopsy was performed using a $16 \mathrm{G}$ needle under ultrasound guidance. Liver samples with a minimum length of $1.5 \mathrm{~cm}$ and at least 7 complete portal tracts were fixed in $10 \%$ formalin, embedded in paraffin, and stained with HE, Masson's trichrome and reticulin staining for histological analysis [20]. Liver histology was analyzed by two experienced pathologists who were blinded to other clinical and laboratory data and classified according to the Scheuer scoring system [21] as follows: S0 (no fibrosis), S1 (mild fibrosis without septa), S2 (moderate fibrosis with few septa), S3 (severe fibrosis with numerous septa without cirrhosis), and S4 (cirrhosis). In this study, liver fibrosis stage S0-S2 was defined as mild/moderate fibrosis, and S3-S4 was defined as advanced fibrosis.

\section{Laboratory data}

Fasting blood samples were obtained within a week of liver biopsy. Platelets and other blood cells were counted using a Sysmex-XT 4000i automated hematology analyzer. The international normalized ratio (INR) and other coagulation indexes were measured using a STAR Max automatic coagulation analyzer. Alanine transaminase (ALT), aspartate aminotransferase (AST), alkaline phosphatase (ALP), $\gamma$-glutamyl transferase (GGT), hyaluronic acid, laminin, $\mathrm{N}$-terminal propeptide of type III procollagen (PIIINP), type IV collagen, and other serum biochemical parameters were measured using an Architect C16000 automatic biochemical analysis system.

\section{Formulas}

The formulas for APRI, FIB-4, and GPR are as follows: $\mathrm{APRI}=(\mathrm{AST}(\mathrm{U} / \mathrm{L}) / \mathrm{ULN}$ of $\mathrm{AST}) /$ platelet count $\left(10^{9} / \mathrm{L}\right) \times 100$; FIB- $4=($ age $($ years $) \times$ AST $(\mathrm{U} / \mathrm{L})) /($ platelet count $\left.\left(10^{9} / \mathrm{L}\right) \times(\mathrm{ALT}(\mathrm{U} / \mathrm{L}))^{1 / 2}\right) ; \mathrm{GPR}=(\mathrm{GGT}(\mathrm{U} / \mathrm{L}) / \mathrm{ULN}$ of GGT)/platelet count $\left(10^{9} / \mathrm{L}\right) \times 100$.

\section{Statistical analysis}

Statistical analysis was performed using IBM SPSS Statistics version 26.0 (SPSS Inc., Chicago, USA) and R 4.0.2 (http://www.R-project.org). Continuous variables are expressed as the mean \pm standard deviation or median (interquartile range, IQR) and compared using the Student's $t$-test (for nomal distribution continuous variables) or independent Mann-Whitney $U$-test (for non-nomal distribution continuous variables). Categorical variables 
are expressed as proportions and compared by the chisquare test. Logistic regression models were used to assess the correlation between variables and liver fibrosis. A nomogram for evaluating liver fibrosis was established based on the results of the multivariate analysis and by using the rms package in $\mathrm{R}$. The predictive accuracy of the nomogram was assessed by calibration. The performances of the established nomogram and other noninvasive markers for predicting liver fibrosis were assessed by receiver operating characteristic (ROC) curve analyses. Delong $\mathrm{Z}$ test was used to compare the AUROC of the serum models. Decision curve analysis (DCA) was used to further evaluate the predictive performances. A twosided $\mathrm{P}<0.05$ was considered statistically significant.

\section{Results}

\section{Baseline demographic and clinical characteristics}

The demographic and clinical characteristics of the studied patients with chronic liver disease are described in Table 1. Overall, the majority of patients suffered from chronic hepatitis B, and there were no significant differences in various clinical parameters between the training set and validation set. Of the training set, 238 patients had biopsy-proven fibrosis S0-S2, and 189 patients had fibrosis S3-S4. ALT, AST, ALP, GGT, DBil, globulin, total bile acid, INR, prothrombin time, APTT, fibrinogen, and thrombin time levels were significantly higher in subjects with fibrosis stages S3-S4 than in subjects with fibrosis stages S0-S2. The levels of albumin, prealbumin, platelet counts, and neutrophil counts were significantly lower in patients with stages S3-S4. In addition, compared with patients with stages S0-S2, the values of biochemical scores significantly increased in patients with stage S3-S4.

\section{Identification of predictive factors for advanced liver fibrosis and cirrhosis}

By using logistic regression analysis, we identified variables related to advanced liver fibrosis and cirrhosis. The presence of advanced liver fibrosis (S3-S4) was associated with AST, ALP, TBil, DBil, cholinesterase, albumin, globulin, prealbumin, total bile acid, TC, TG, LDL, prothrombin time, INR, thrombin time, RBC, platelet count, neutrophil count, hyaluronic acid, cholylglycine, laminin, PIIINP, and type IV collagen. Multivariable analysis identified INR $(P=0.003$, odds ratio $[\mathrm{OR}]=1.58,95 \%$ confidence interval $[\mathrm{CI}]=1.17-2.15)$, platelets $(P<0.001$, $\mathrm{OR}=0.99,95 \% \mathrm{CI}=0.98-1.00)$, and PIIINP $(P<0.001$, $\mathrm{OR}=1.06,95 \% \mathrm{CI}=1.04-1.09)$ as independent predictors of advanced liver fibrosis.

The presence of cirrhosis (S4) was associated with AST, ALP, TBil, DBil, cholinesterase, albumin, globulin, prealbumin, total bile acid, TC, TG, LDL, PT, INR, fibrinogen, thrombin time, WBC, platelet count, neutrophil count, hyaluronic acid, cholylglycine, laminin, PIIINP, and type IV collagen. Multivariable analysis identified INR $(P=0.001, \quad \mathrm{OR}=1.85, \quad 95 \% \quad \mathrm{CI}=1.29-2.15)$, platelets $(P<0.001, \mathrm{OR}=0.97,95 \% \mathrm{CI}=0.96-0.98)$, and PIIINP $(P<0.001, \mathrm{OR}=1.02,95 \% \mathrm{CI}=1.01-1.04)$ as independent predictors of cirrhosis.

\section{Predictive nomogram construction and calibration in the training cohort}

The predictive nomograms that combined all significant independent predictive factors for advanced fibrosis and cirrhosis in the training cohort are shown in Fig. 1. The independent factors included platelet counts, INR, and PIIINP. A sum score could be calculated as the total scores of related predictors and referred to the probability of advanced fibrosis or cirrhosis in the basal axis. For example, in a patient whose INR was 1.1, their platelet count was $200 \times 10^{9} / \mathrm{L}$, PIIINP was $58 \mathrm{ng} / \mathrm{ml}$, total points scored was 0.42 , and advanced fibrosis probability was approximately $70 \%$; in a patient whose INR was 1.1 , platelet count was $200 \times 10^{9} / \mathrm{L}$, their PIIINP was $140 \mathrm{ng} /$ $\mathrm{ml}$, total points scored was 0.88 , and cirrhosis probability was approximately $40 \%$.

The calibration plot for the probability of advanced fibrosis showed optimal agreement between the prediction by the nomogram S3S4 and the actual observation (Fig. 2a). Remarkably, the calibration plot for the probability of cirrhosis showed good consistency between the nomogram S4 prediction and actual observation (Fig. 2b).

\section{Comparison of the predictive accuracy between nomograms and noninvasive markers in the training cohort and validation cohort}

The established nomograms displayed better accuracy in predicting advanced fibrosis and cirrhosis. The ROC curves for our nomograms, APRI, FIB-4, and GPR are shown in training set (Fig. 3) and validation set (Fig. 4), respectively. In the training set, for discriminating advanced fibrosis, Nomogram S3S4 had the highest areas under the ROC curve (AUROC) (0.83, sensitivity $78.9 \%$ and specificity $79.4 \%$ ) compared with APRI (0.71, sensitivity $81.5 \%$ and specificity $51.7 \%)$, FIB- 4 ( 0.68 , sensitivity $38.6 \%$ and specificity $90.6 \%$ ), and GPR (0.74, sensitivity $73.2 \%$ and specificity $69.3 \%$ ). When discriminating cirrhosis, nomogram S4 had the best AUROC (0.88, sensitivity $77.3 \%$ and specificity $85.2 \%$ ) compared with APRI (0.74, sensitivity $78.2 \%$ and specificity $57.8 \%)$, FIB-4 (0.78, sensitivity $53.7 \%$ and specificity $90.1 \%)$, and GPR $(0.79$, sensitivity $67.3 \%$ and specificity $80.8 \%$ ) (Table 2 ). Similarly, in the validation set, compared to the other three serum indexes, nomogram S3S4 had the highest AUROC 
Table 1 Clinical characteristics of studied patients with chronic liver disease

\begin{tabular}{|c|c|c|c|c|c|c|}
\hline \multirow[t]{2}{*}{ Variables } & \multicolumn{3}{|l|}{ Total $(n=701)$} & \multicolumn{3}{|c|}{ Training cohort $(n=427)$} \\
\hline & Training set $(n=427)$ & Validation set $(\mathrm{n}=274)$ & $P$ value & S0-S2 (n=238) & S3-S4 $(n=189)$ & $P$ value \\
\hline Age, years & $37(31-46)$ & $36(30-46)$ & 0.171 & $38(31-46)$ & $37(31-45)$ & 0.488 \\
\hline Male, n (\%) & $284(66.5)$ & $181(66.1)$ & 0.902 & $158(66.4)$ & $129(68.3)$ & 0.683 \\
\hline \multicolumn{7}{|l|}{ Aetiology } \\
\hline Chronic hepatitis B, n (\%) & $263(61.6)$ & $220(80.3)$ & $<0.001$ & $135(56.8)$ & $128(67.7)$ & 0.020 \\
\hline Chronic hepatitis C, n (\%) & $40(9.4)$ & $8(3.0)$ & 0.001 & $20(8.4)$ & $20(10.7)$ & 0.552 \\
\hline NAFLD, n (\%) & $43(10.1)$ & $30(10.9)$ & 0.710 & $31(13.0)$ & $12(6.3)$ & 0.023 \\
\hline Alcoholic liver disease, n (\%) & $30(7.0)$ & $5(1.8)$ & 0.002 & $25(10.5)$ & $5(2.6)$ & 0.002 \\
\hline Autoimmune hepatitis, n (\%) & $51(11.9)$ & $11(4.0)$ & $<0.001$ & $27(11.3)$ & $24(12.7)$ & 0.668 \\
\hline \multicolumn{7}{|l|}{ Blood parameters } \\
\hline$A L T, U / L$ & $56.00(31.00-123.00)$ & $67.00(28.00-148.50)$ & 0.320 & $50.50(23.00-112.50)$ & $66.00(38.00-143.50)$ & 0.002 \\
\hline AST, U/L & $41.00(26.00-76.00)$ & $41.00(24.00-96.25)$ & 0.378 & $33.50(22.00-62.25)$ & $50.00(31.00-89.50)$ & $<0.001$ \\
\hline$A L P, U / L$ & $77.00(64.00-93.00)$ & 75.00 (63.00-99.00) & 0.574 & $72.00(59.75-86.25)$ & $85.00(67.00-101.50)$ & $<0.001$ \\
\hline $\mathrm{GGT}, \mathrm{U} / \mathrm{L}$ & $35.00(19.00-67.00)$ & $35.00(19.00-86.00)$ & 0.500 & $26.00(16.00-47.00)$ & $49.00(28.50-85.00)$ & $<0.001$ \\
\hline TBil, $\mu \mathrm{mol} / \mathrm{L}$ & $15.05(11.10-19.85)$ & $14.60(10.08-20.28)$ & 0.800 & $14.40(11.15-18.85)$ & $15.70(11.10-21.70)$ & 0.054 \\
\hline DBil $\mu \mathrm{mol} / \mathrm{L}$ & $5.50(4.10-7.50)$ & $5.25(3.70-8.13)$ & 0.414 & $5.25(4.00-6.80)$ & $6.10(4.60-8.70)$ & $<0.001$ \\
\hline Albumin, $g / L$ & $42.00(39.20-44.50)$ & $42.20(39.88-44.53)$ & 0.403 & $42.80(40.20-45.25)$ & $41.00(38.20-43.75)$ & $<0.001$ \\
\hline Globulin, g/L & $29.00(26.00-32.00)$ & $27.00(24.00-30.00)$ & $<0.001$ & $29.00(26.00-32.00)$ & $30.00(26.50-33.00)$ & 0.034 \\
\hline Prealbumin, $\mathrm{g} / \mathrm{L}$ & $200.20(151.00-249.35)$ & $209.00(145.00-258.00)$ & 0.389 & $217.00(170.25-269.00)$ & $170.00(127.50-218.00)$ & $<0.001$ \\
\hline Total bile acid, $\mu \mathrm{mol} / \mathrm{L}$ & $8.75(4.20-15.60)$ & $9.70(5.50-20.75)$ & 0.003 & $6.70(3.50-12.00)$ & $11.80(6.60-20.60)$ & $<0.001$ \\
\hline $\mathrm{FBG}, \mathrm{mmol} / \mathrm{L}$ & $4.79(4.49-5.21)$ & $4.90(4.57-5.32)$ & 0.060 & $4.82(4.57-5.24)$ & $4.74(4.46-5.15)$ & 0.032 \\
\hline $\mathrm{TC}, \mathrm{mmol} / \mathrm{L}$ & $4.14(3.66-4.83)$ & $4.07(3.57-4.67)$ & 0.106 & $4.27(3.79-4.89)$ & $3.98(3.50-4.68)$ & 0.001 \\
\hline $\mathrm{TG}, \mathrm{mmol} / \mathrm{L}$ & $0.93(0.71-1.24)$ & $1.00(0.75-1.40)$ & 0.022 & $0.96(0.75-1.31)$ & $0.88(0.70-1.11)$ & 0.006 \\
\hline $\mathrm{HDL}, \mathrm{mmol} / \mathrm{L}$ & $1.32(1.06-1.64)$ & $1.32(1.06-1.54)$ & 0.278 & $1.34(1.04-1.63)$ & $1.31(1.08-1.67)$ & 0.930 \\
\hline LDL mmol/L & $2.60(2.11-3.18)$ & $2.45(2.02-3.07)$ & 0.017 & $2.70(2.27-3.32)$ & $2.40(2.01-2.91)$ & $<0.001$ \\
\hline Urea, mmol/L & $314.00(257.00-370.18)$ & & & $314.80(248.05-381.50)$ & $312.55(268.38-356.08)$ & 0.682 \\
\hline Creatinine, $\mu \mathrm{mol} / \mathrm{L}$ & $65.25(55.17-75.10)$ & $68.10(56.30-77.20)$ & 0.124 & $63.75(55.50-74.50)$ & $67.15(54.50-75.73)$ & 0.330 \\
\hline Prothrombin time, s & $13.80(13.20-14.40)$ & $13.60(13.00-14.30)$ & 0.021 & $13.50(13.10-14.00)$ & $14.10(13.50-15.00)$ & $<0.001$ \\
\hline INR & $1.05(1.00-1.12)$ & $1.05(0.99-1.10)$ & 0.075 & $1.03(0.99-1.08)$ & $1.09(1.03-1.17)$ & $<0.001$ \\
\hline APTT, s & $38.60(36.30-41.20)$ & 38.65 (35.83-41.78) & 0.877 & $37.70(35.60-40.00)$ & $39.80(37.30-42.20)$ & $<0.001$ \\
\hline Fibrinogen, g/L & $2.50(2.17-2.79)$ & $2.38(2.04-2.64)$ & 0.001 & $2.59(2.25-2.90)$ & $2.36(2.09-2.62)$ & $<0.001$ \\
\hline Thrombin time, s & $17.70(17.00-18.50)$ & $17.80(17.00-18.70)$ & 0.508 & $17.45(16.90-18.23)$ & $18.10(17.30-18.80)$ & $<0.001$ \\
\hline WBC count, $\times 10^{9} / \mathrm{L}$ & $5.28(4.23-6.27)$ & $5.24(4.33-6.25)$ & 0.595 & $5.41(4.39-6.45)$ & $5.14(4.12-6.14)$ & 0.057 \\
\hline RBC count, $\times 10^{9} / \mathrm{L}$ & $4.65(4.26-5.01)$ & $4.56(4.12-4.93)$ & 0.004 & $4.75(4.35-5.06)$ & $4.54(4.12-4.95)$ & 0.003 \\
\hline Platelet count, $\times 10^{9} / \mathrm{L}$ & $159.00(130.00-195.00)$ & $152.50(122.00-185.25)$ & 0.066 & $177.00(150.00-207.25)$ & $140.00(98.50-171.50)$ & $<0.001$ \\
\hline Hemoglobin (g/L) & $145.00(132.00-156.00)$ & $141.00(128.00-154.00)$ & 0.014 & $147.00(133.75-157.00)$ & $143.00(129.00-155.00)$ & 0.118 \\
\hline Neutrophils count, $\times 10^{9} / \mathrm{L}$ & $2.84(2.18-3.58)$ & $2.69(2.07-3.42)$ & 0.083 & $2.98(2.35-3.65)$ & $2.62(1.98-3.53)$ & 0.002 \\
\hline \multicolumn{7}{|l|}{ Biochemical scores } \\
\hline Hyaluronic, ng/ml & $61.35(44.43-94.09)$ & $74.98(46.37-117.55)$ & 0.004 & $52.18(40.58-73.89)$ & $84.50(53.87-140.50)$ & $<0.001$ \\
\hline Cholyglycine, ug/ml & $2.65(1.48-5.26)$ & $2.30(1.38-5.79)$ & 0.365 & $1.98(1.11-4.46)$ & $3.65(2.03-7.49)$ & $<0.001$ \\
\hline Laminin, ng/ml & $19.17(10.01-30.40)$ & $31.35(23.48-41.08)$ & $<0.001$ & $14.96(8.25-23.47)$ & $25.46(16.03-38.31)$ & $<0.001$ \\
\hline PIIINP, ng/ml & $26.50(19.06-39.64)$ & $27.45(21.99-37.71)$ & 0.065 & $21.10(16.43-27.57)$ & $38.06(27.16-53.96)$ & $<0.001$ \\
\hline Type IV collagen, ng/ml & $26.11(19.05-37.80)$ & $25.90(20.78-36.35)$ & 0.410 & $21.60(15.67-26.96)$ & $37.10(26.77-53.11)$ & $<0.001$ \\
\hline APRI & $0.68(0.38-1.37)$ & $0.75(0.38-1.83)$ & 0.095 & $0.49(0.29-0.94)$ & $0.97(0.58-1.82)$ & $<0.001$ \\
\hline FIB-4 & $1.33(0.92-2.15)$ & $1.32(0.95-2.26)$ & 0.444 & $1.17(0.85-1.66)$ & $1.69(1.07-3.00)$ & $<0.001$ \\
\hline GPR & $0.23(0.11-0.50)$ & $0.55(0.26-1.33)$ & $<0.001$ & $0.15(0.08-0.29)$ & $0.38(0.21-0.81)$ & $<0.001$ \\
\hline
\end{tabular}

NAFLD, nonalcoholic fatty liver disease; ALT, alanine transaminase; AST, aspartate aminotransferase; ALP, alkaline phosphatase; GGT, $\gamma$-glutamyl transpeptadase; TBil, total bilirubin; DBil, direct bilirubin; FBG, fasting blood glucose; TC, total cholesterol; TG, triglyceride; LDC, low-density lipoprotein; HDL, high-density lipoprotein; INR, international normalized ratio; APTT, activated partial thromboplastin time 


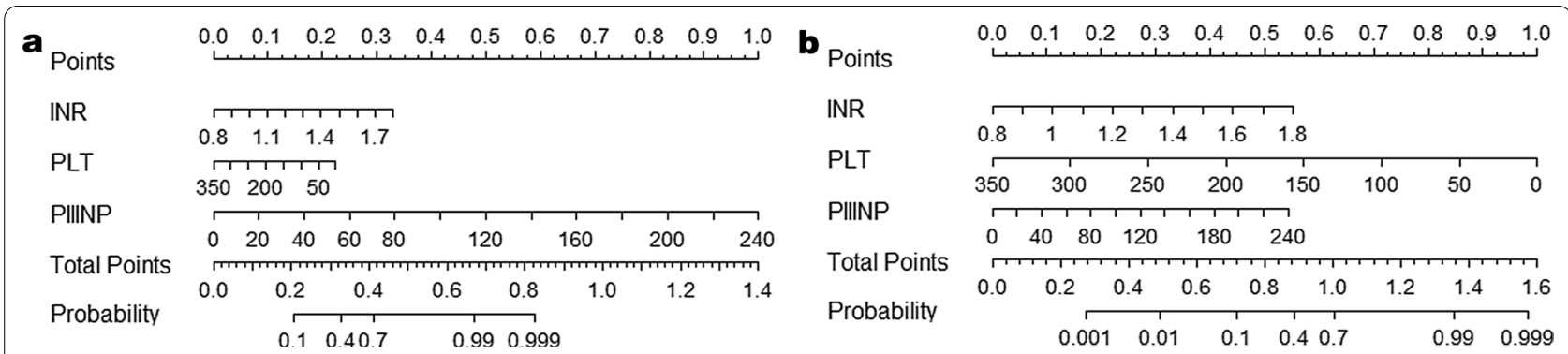

Fig. 1 Liver fibrosis Nomograms in training set. The nomogramS3S4 (a) and the nomogramS4 (b) were constructed for evaluation of advanced fibrosis and cirrhosis, respectively. Each variable is assigned a point on the top axis by drawing a line upward. The sum of these numbers is located on the Total Points axis, and a line is drawn downwards to the Probability axis to identify the likelihood of advanced fibrosis and cirrhosis
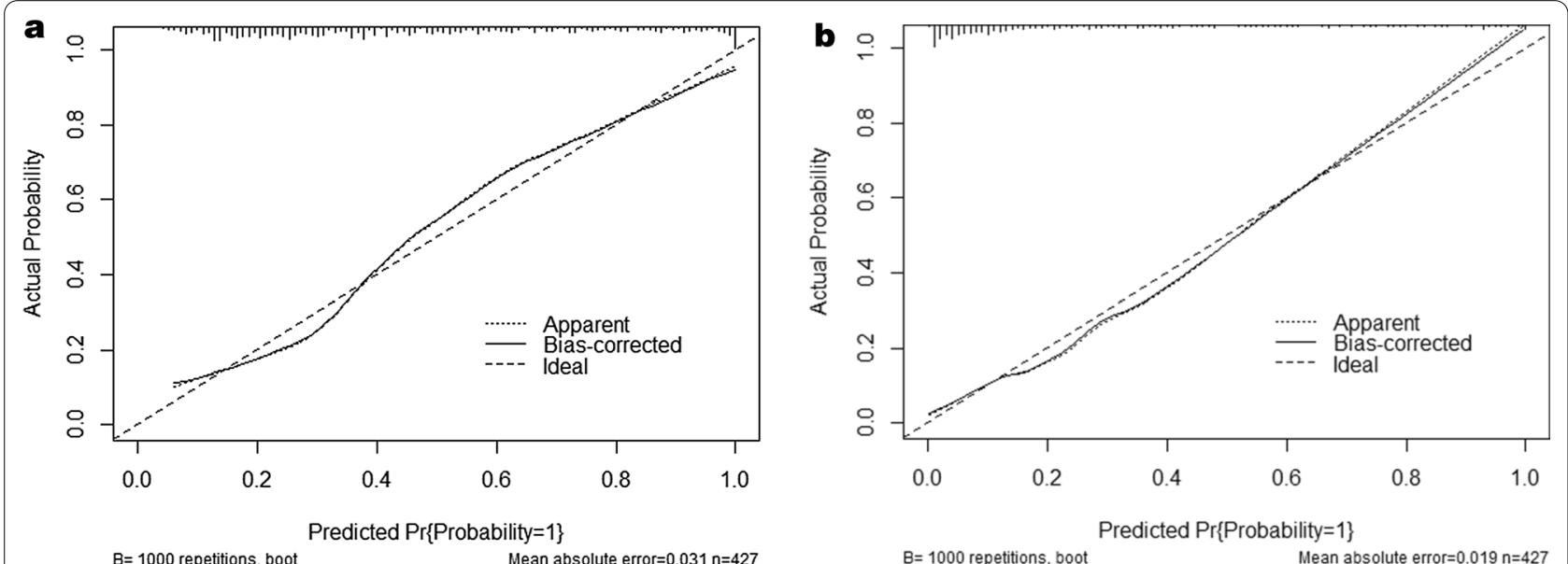

Fig. 2 Liver fibrosis calibration curves in training set. The calibration curves for predicting advanced fibrosis (a) and cirrhosis (b) in chronic liver disease patients. Nomograms-predicted probability of liver fibrosis stages is plotted on the $x$-axis, and actual probability is plotted on the $y$-axis

(0.86, sensitivity $70.0 \%$ and specificity $87.1 \%)$ for predicting advanced fibrosis. nomogram $\mathrm{S} 4$ had the best AUROC (0.88, sensitivity $54.0 \%$ and specificity $95.9 \%)$ for predicting cirrhosis (Table 3). These results suggest that the nomograms were useful predictors of advanced fibrosis and cirrhosis for patients with chronic liver disease.

\section{DCA for clinical utility of the established nomograms}

In addition, we conducted DCA to further investigate the clinical application values of the nomograms in predicting advanced fibrosis and cirrhosis. In the training set, DCA revealed that from a threshold probability of $10-80 \%$, the application of nomogram S3S4 to predict advanced fibrosis risk increased the benefit considerably more than the other three scores (Fig. 5a). In particular, employing nomogram S4 allowed us to obtain much more net benefit at any threshold probability (Fig. 5b). As we expected, the DCAs of the validation set and our nomograms also showed a better net benefit with a wide range of threshold probabilities and better performances for predicting advanced fibrosis and cirrhosis than APRI, FIB-4, and GPR (Fig. 5c, d).

\section{Discussion}

Although all international guidelines recommend early treatment for patients with chronic liver disease, it is estimated that two-thirds of patients with cirrhosis are diagnosed in time when they have liver-related complications, compromising their short-term prognosis. In our study, the INR, PLT, and PIIINP were independent risk factors for progressive liver fibrosis in patients with chronic liver disease [22]. This indicates that there is an urgent need for simple and accurate tools to identify the subgroup of chronic liver disease patients who require special treatment by professional physicians. We subsequently constructed noninvasive nomograms that incorporated the three indexes to predict advanced fibrosis and cirrhosis in chronic liver disease patients to assess the progression of liver fibrosis in a noninvasive procedure in chronic liver disease patients. The established nomograms are convenient, useful and user-friendly in clinical practice. 

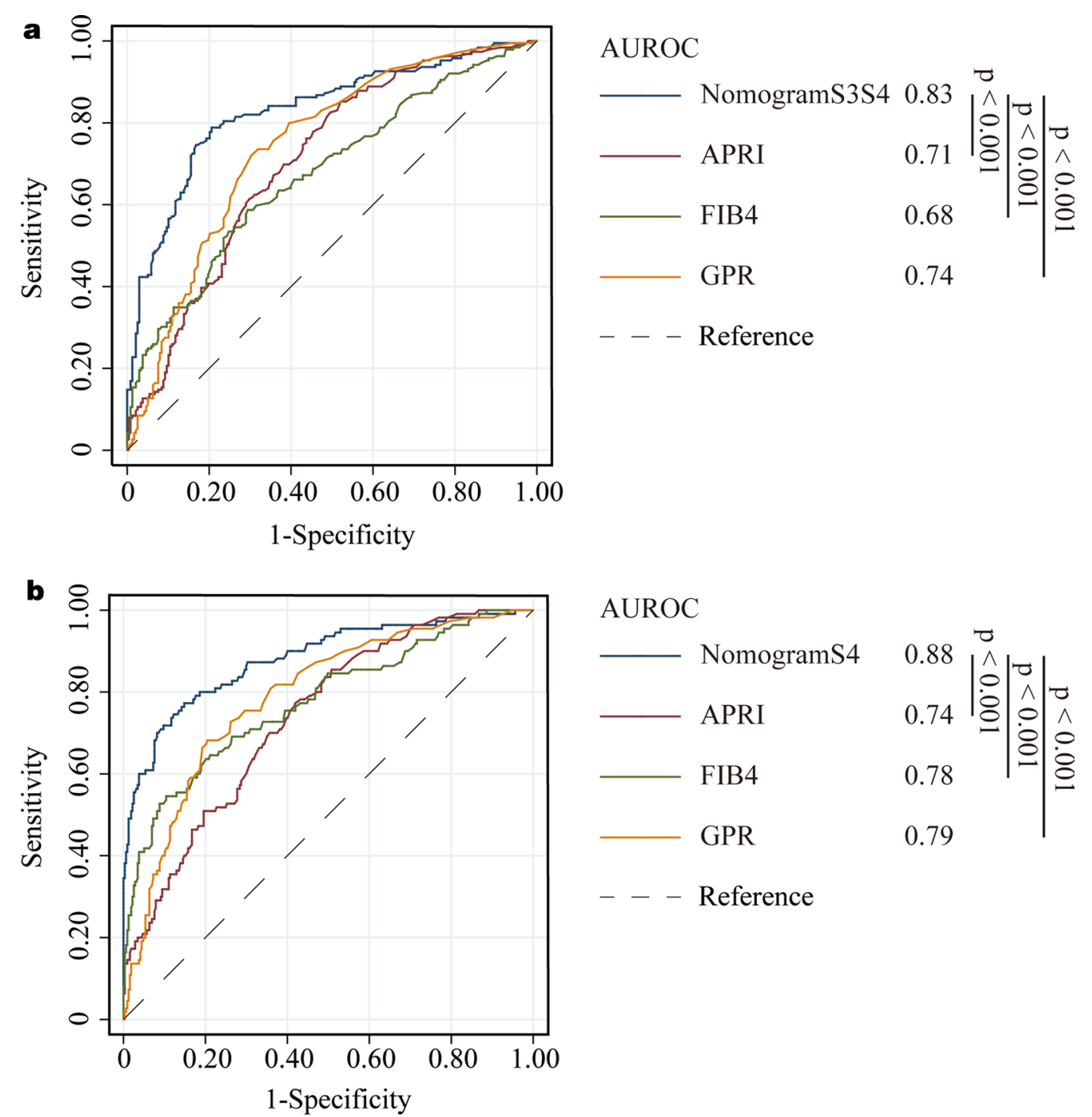

AUROC

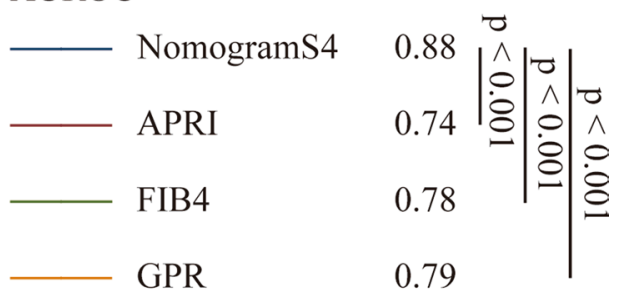

- - - Reference

INR is a known parameter often used in the assessment of liver fibrosis. Sterlin et al. [23] reported that the INR was an independent predictor for liver fibrosis and that its level correlated directly with liver function. Cross et al. [24] reported that the INR level correlated with liver fibrosis and was used as a parameter in the Kings score, which was helpful to diagnose liver fibrosis. Furthermore, Takaki et al. [25] reported that the INR correlates independently and significantly with significant liver fibrosis in hepatitis $\mathrm{C}$ patients and used it to develop the VIA index to measure liver fibrosis stage.

The process of liver fibrosis is thought to be caused by excessive production of extracellular matrix (ECM). In the liver type III collagen mainly occurs [26]. During the synthesis of type III collagen, PIIINP is detached from procollagen type III [27], so fibrogenesis results in the release of ECM fragments into the blood. Thus, circulating levels of PIIINP can be a biomarker of hepatic fibrogenesis $[27,28]$. In some observational studies, plasma PIIINP levels have been shown to be strongly associated with liver fibrosis in patients with chronic liver disease [29-32]. The best predictive value was reported, with an $A U R O C=0.87$ distinguishing fibrosis and cirrhosis from normal liver or steatosis in 45 cases with alcoholic liver disease [31]. A small study demonstrated that the combination of genetic variations and enhanced liver fibrosis (ELF) indexes (tissue inhibitor of matrix metalloproteinase-1, hyaluronic acid, and PIIINP) could predict 


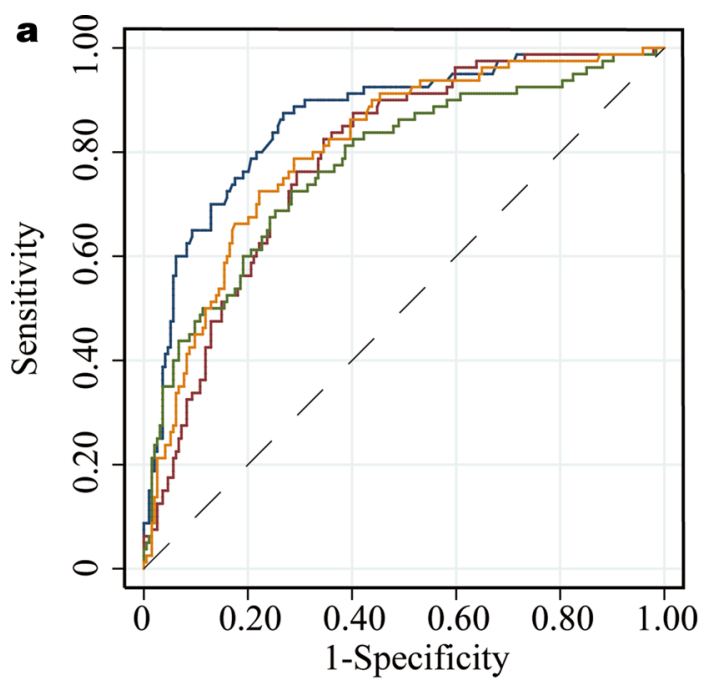

AUROC

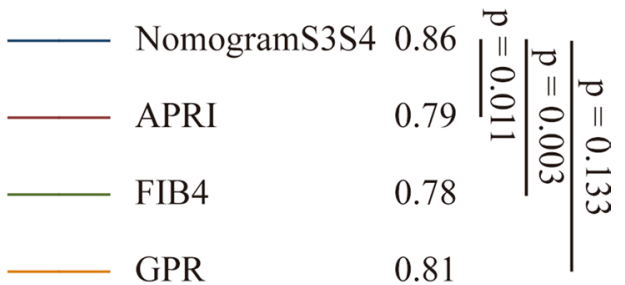

- - Reference

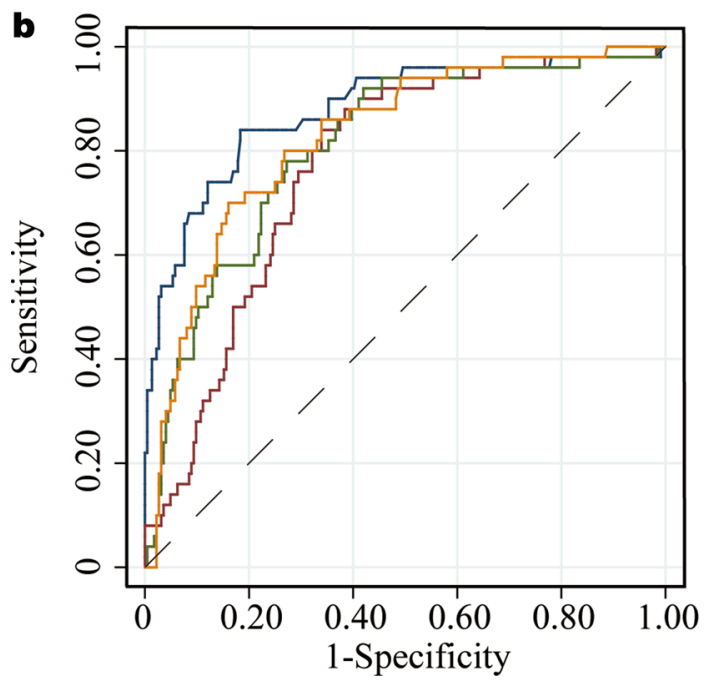

AUROC

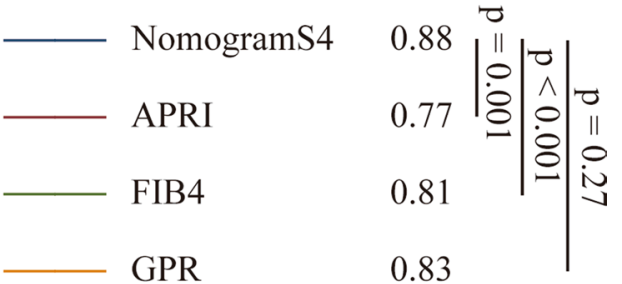

- - Reference

Fig. 4 Area under receiver operating characteristic (ROC) comparison of Nomograms, APRI, FIB-4, and GPR in validation set. a ROC comparison for predicting advanced fibrosis; $\mathbf{b} \mathrm{ROC}$ comparison for predicting cirrhosis

the progression of fibrosis in 56 patients with mid-liver fibrosis over 5 years [33]. Another study showed that compared with other clinical scores, the ELF test for 457 HCV patients could better predict morbidity and mortality after 7 years of follow-up [34]. Nielsen et al. [35] developed the PRO-C3 assay (a neoepitope-specific serum ELISA for type III procollagen) that could accurately predict advanced fibrosis in patients with nonalcoholic fatty liver disease (NAFLD). Recently, a PRO-C3-based fibrosis algorithm that included age, diabetes, PRO-C3, and platelet count (ADAPT) was developed to more accurately identify advanced fibrosis in patients with NAFLD compared to APRI, FIB-4, and NAFLD fibrosis score [36]. One possible explanation for the increased PIIINP level in advanced fibrosis is that ECM remodeling is an attempt to repair damaged tissue in response to liver injury. If liver damage is extensive, ECM components and collagen synthesis will be increased.

Several new noninvasive methods for the prediction and assessment of liver fibrosis have recently become available. In this study, the predictive power of the established nomograms in the assessment of liver fibrosis was excellent in both the training group and validation group and was significantly higher than that of the APRI, FIB-4, and GPR. The nomograms provided better sensitivity and specificity than the other three markers for staging advanced fibrosis and cirrhosis. Moreover, in the DCA analysis, a new analysis that visually 
Table 2 Predictive performances of nomograms, APRI, FIB-4, and GPR for advanced fibrosis (S3-S4) and cirrhosis (S4) (Training cohort)

\begin{tabular}{llllll}
\hline Indexes & AUROC (95\%Cl) & $P$ & Cutoff & Se (\%) & Sp (\%) \\
\hline $\begin{array}{l}\text { S3-S4 } \\
\begin{array}{c}\text { Nomo- } \\
\text { gramS3S4 }\end{array}\end{array}$ & $0.83(0.79-0.87)$ & $<0.0001$ & 0.41 & 78.9 & 79.4 \\
APRI & $0.71(0.66-0.75)$ & $<0.0001$ & 0.51 & 81.5 & 51.7 \\
FIB-4 & $0.68(0.60-0.70)$ & $<0.0001$ & 1.48 & 58.7 & 70.6 \\
GPR & $0.74(0.70-0.79)$ & $<0.0001$ & 0.23 & 73.2 & 69.3 \\
S4 & & & & & \\
NomogramS4 & $0.88(0.85-0.91)$ & $<0.0001$ & 0.91 & 77.3 & 85.2 \\
APRI & $0.74(0.70-0.79)$ & $<0.0001$ & 0.66 & 78.2 & 57.8 \\
FIB-4 & $0.78(0.73-0.81)$ & $<0.0001$ & 2.34 & 54.6 & 89.6 \\
GPR & $0.79(0.75-0.83)$ & $<0.0001$ & 0.38 & 67.3 & 80.8 \\
\hline
\end{tabular}

AUROC, area under ROC; Se, sensitivity; Sp, specificity

Table 3 Predictive performances of nomograms, APRI, FIB-4, and GPR for advanced fibrosis (S3-S4) and cirrhosis (S4) Validation cohort)

\begin{tabular}{llllll}
\hline Indexes & AUROC (95\%Cl) & $P$ & Cutoff & Se (\%) & Sp (\%) \\
\hline $\begin{array}{llllll}\text { S3-S4 } \\
\quad \text { Nomo- }\end{array} \quad 0.86(0.82-0.90)$ & $<0.0001$ & 0.41 & 70.0 & 87.1 \\
$\quad$ gramS3S4 & & & & & \\
APRI & $0.79(0.73-0.83)$ & $<0.0001$ & 0.51 & 91.2 & 47.0 \\
FIB-4 & $0.78(0.72-0.82)$ & $<0.0001$ & 1.48 & 72.5 & 68.6 \\
GPR & $0.81(0.76-0.85)$ & $<0.0001$ & 0.23 & 97.5 & 28.9 \\
S4 & & & & & \\
NomogramS4 & $0.88(0.83-0.92)$ & $<0.0001$ & 0.91 & 54.0 & 95.9 \\
APRI & $0.77(0.72-0.82)$ & $<0.0001$ & 0.66 & 92.0 & 53.6 \\
FIB-4 & $0.81(0.76-0.85)$ & $<0.0001$ & 2.34 & 58.0 & 84.4 \\
GPR & $0.83(0.78-0.87)$ & $<0.0001$ & 0.38 & 94.0 & 46.4 \\
\hline
\end{tabular}

AUROC, area under ROC; Se, sensitivity; Sp, specificity

predicted the clinical applicability of a model, our nomograms demonstrated a superior benefit across a wider range of threshold probabilities compared with APRI, FIB-4, and GPR.

APRI and FIB-4 are the two widely studied noninvasive methods for assessing liver fibrosis stage.
Teshale et al. [37] assessed the predictive values of APRI and FIB-4 in a large cohort of chronic hepatitis $B$ patients and demonstrated that APRI and FIB-4 distinguished significant liver fibrosis with good sensitivity and specificity. The WHO hepatitis B guidelines have recommended APRI to predict liver fibrosis stage in resource-limited countries [12]. In a metaanalysis pooling $4266 \mathrm{HCV}$ patients from 22 studies, the AUROCs of ARPI for diagnosing significant fibrosis and cirrhosis were 0.76 and 0.86 , respectively [38]. However, another meta-analysis indicated that APRI and FIB-4 had only moderate sensitivity and accuracy for diagnosis of liver fibrosis in chronic hepatitis B patients, which suggested they were not ideal substitutes for liver biopsy [39]. Regarding NAFLD, recent studies showed that APRI could better distinguish fibrosis stage F2-3 from F0-1 than FIB-4 [40, 41]. The GPR developed by Lemoine et al. was shown to be more accurate than the APRI and FIB-4 [42]. Additionally, GPR is reported to predict significant fibrosis and cirrhosis excellently in a large cohort of chronic hepatitis B Gambian patients using FibroScan measures as a reference [43]. However, it did show a controversial advantage in several Chinese cohorts $[8,44]$.

Our study has several limitations. First, this is a retrospective study in a single center, so it should be validated with more patients from other centers. Second, further prospective studies are needed to investigate whether the established nomograms had disease-specific cutoffs to distinguish stages of liver fibrosis.

\section{Conclusion}

In summary, these results suggest that serum INR, PLT, and PIIINP are independent predictors of advanced liver fibrosis and cirrhosis. Furthermore, the nomogram S3-4 and nomogram S4 established based on the three parameters demonstrate superior clinical usefulness. Although future validation in a large cohort is needed, these established nomograms in a noninvasive manner could be promising procedures for staging liver fibrosis, especially in resource-limited regions. 


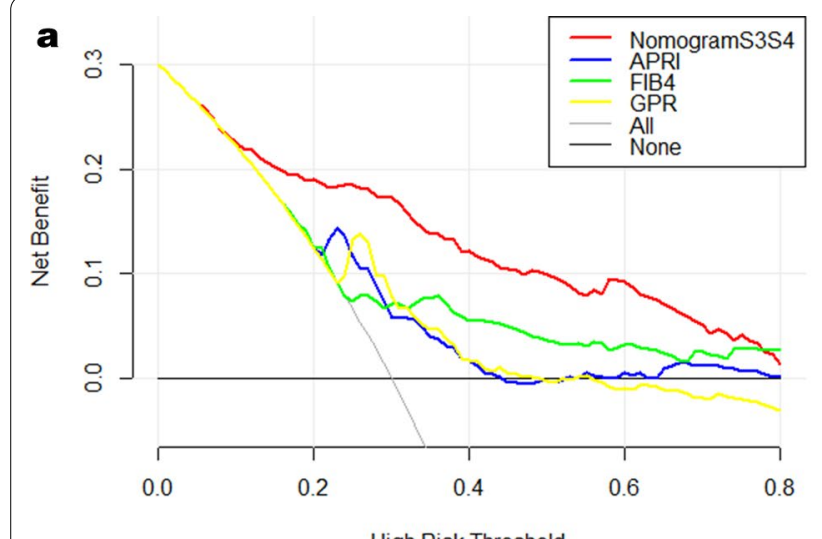

High Risk Threshold

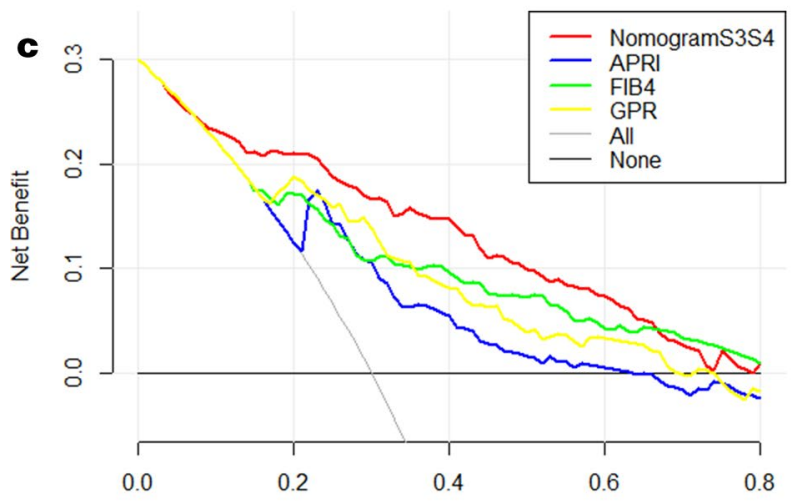

High Risk Threshold
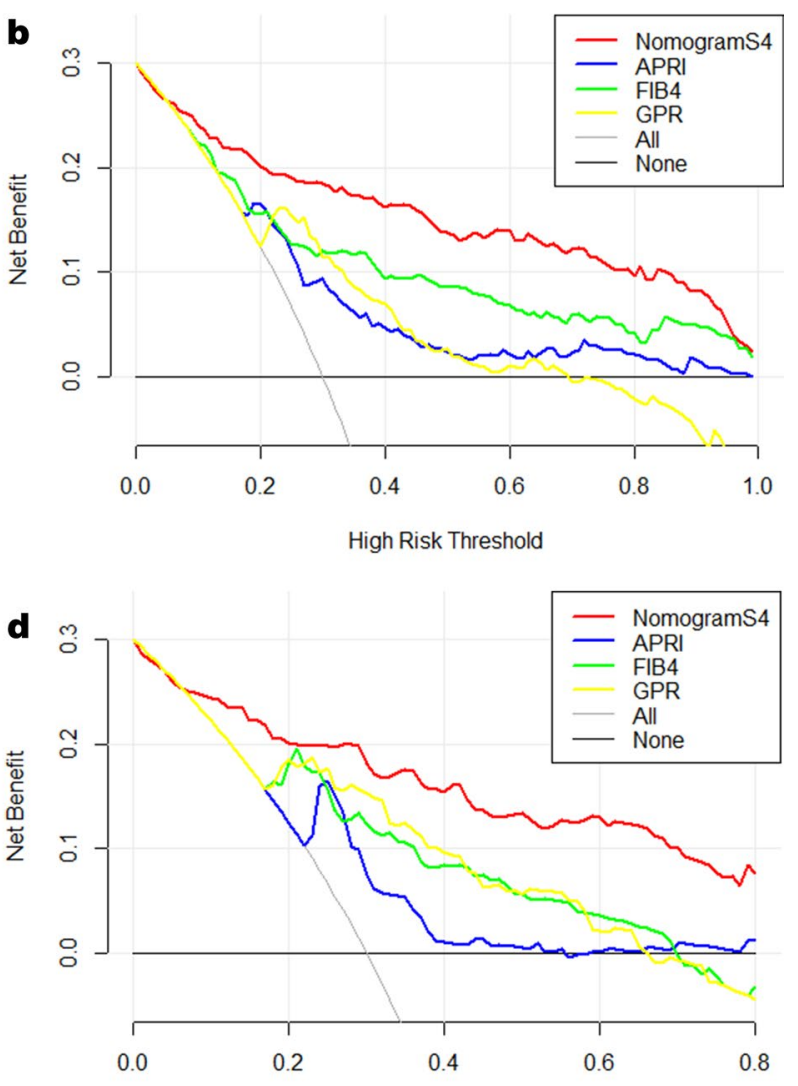

High Risk Threshold

Fig. 5 Liver fibrosis decision curve analysis. Decision curve analysis depict the clinical net benefit. NomogramS3S4 is compared with APRI, FIB-4, and GPR for predicting advanced fibrosis in the training set (a); NomogramS4 is compared with APRI, FIB-4, and GPR for predicting cirrhosis in the validation set (b); NomogramS3S4 is compared with APRI, FIB-4, and GPR for predicting advanced fibrosis in the training set (c); NomogramS4 is compared with APRI, FIB-4, and GPR for predicting cirrhosis in the validation set (d). Black line= net benefit when no patient will experience the event; gray line $=$ net benefit when all patients will experience the event. The preferred markers is the marker with the highest net benefit at any given threshold

\section{Abbreviations}

APRI: Aspartate transaminase to platelet ratio; FIB-4: Fibrosis-4 score; GPR: $\gamma$-Glutamyl transpeptidase to platelet ratio; INR: International normalized ration; ALT: Alanine transaminase; AST: Aspartate aminotransferase; ALP: Alkaline phosphatase; GGT: y-Glutamyl transfetase; PIIINP: N-terminal propeptide of type III procollagen; ULN: Upper limit of normal; ROC: Receiver operating characteristics; DCA: Decision curve analysis; AUROC: Areas under ROC; ECM: Extracellular matrix; ELF: Enhanced liver fibrosis indexes; NAFLD: Nonalcoholic fatty liver disease.

\section{Acknowledgements}

Not applicable.

\section{Authors' contributions}

DRR designed the study and wrote the manuscript; ZXL and HD collected and analyzed data; WYB and YL reviewed the statistical data. LXF and LW were involved in critical revision of the paper. YZG and ZZQ approved the final manuscript. All authors have read and approved the manuscript.

\section{Funding}

This work was supported by the "13th Five-year" National Science and Technology Major Project of China (2017ZX10203202). This found was acquired by
Zhanging Zhang. The funding body had no role in the design of the study and collection, analysis, and interpretation of data and in writing the manuscript.

\section{Availability of data and materials}

Datasets of the current study are available from the corresponding authors on reasonable request.

\section{Ethics approval and consent to participate}

The study protocol and informed consent documents were reviewed and approved by the Ethics Committee of Shanghai Public Health Clinical Center, Fudan University (2021-S011-01). All included patients provided written informed consent before participating in this study.

\section{Consent for publication}

Not applicable.

\section{Competing interests}

The authors declare that there is no competing interests.

Received: 23 November 2020 Accepted: 20 April 2021

Published online: 27 April 2021 


\section{References}

1. D'Amico G, Garcia-Tsao G, Pagliaro L. Natural history and prognostic indicators of survival in cirrhosis: a systematic review of 118 studies. J Hepatol. 2006;44(1):217-31

2. Girleanu I, Trifan A, Singeap AM, Stoica OC, Cojocariu C, Stanciu C. Platelet indices and liver fibrosis evaluation in chronic hepatitis C. Rev Med ChirSoc Med Nat lasi. 2016;120(1):55-61.

3. Li CJ, Yang ZH, Lu FG, Shi XL, Liu DL. Clinical significance of fibrotic, haemostatic and endotoxic changes in patients with liver cirrhosis. ActaGastroenterolBelg. 2018;81(3):404-9.

4. Imai H, Kamei H, Onishi Y, Ishizu Y, Ishigami M, Goto H, Ogura Y. Diagnostic usefulness of APRI and FIB-4 for the prediction of liver fibrosis after liver transplantation in patients infected with hepatitis $C$ virus. Transplant Proc. 2018;50(5):1431-6.

5. Stockdale AJ, Phillips RO, Geretti AM, Group HS. The gamma-glutamyl transpeptidase to platelet ratio (GPR) shows poor correlation with transient elastography measurements of liver fibrosis in HIV-positive patients with chronic hepatitis B in West Africa. Response to: 'The gamma-glutamyl transpeptidase to platelet ratio (GPR) predicts significant liver fibrosis and cirrhosis in patients with chronic HBV infection in West Africa' by Lemoine et al. Gut. 2016;65(5):882-884.

6. Wai CT, Greenson JK, Fontana RJ, Kalbfleisch JD, Marrero JA, Conjeevaram HS, Lok AS. A simple noninvasive index can predict both significant fibrosis and cirrhosis in patients with chronic hepatitis C. Hepatology. 2003;38(2):518-26.

7. Huang D, Lin T, Wang S, Cheng L, Xie L, Lu Y, Chen M, Zhu L, Shi J. The liver fibrosis index is superior to the APRI and FIB-4 for predicting liver fibrosis in chronic hepatitis B patients in China. BMC Infect Dis. 2019;19(1):878.

8. Huang R, Wang G, Tian C, et al. Gamma-glutamyl-transpeptidase to platelet ratio is not superior to APRI, FIB-4 and RPR for diagnosing liver fibrosis in CHB patients in China. Sci Rep. 2017;7(1):8543.

9. Huang ZL, Chen XP, Zhao QY, Zheng YB, Peng L, Gao ZL, Zhao ZX. An albumin, collagen IV, and longitudinal diameter of spleen scoring system superior to APRI for assessing liver fibrosis in chronic hepatitis $B$ patients. Int J Infect Dis. 2015;31:18-22.

10. Friedrich-Rust M, Ong MF, Martens S, Sarrazin C, Bojunga J, Zeuzem S, Herrmann E. Performance of transient elastography for the staging of liver fibrosis: a meta-analysis. Gastroenterology. 2008;134(4):960-74.

11. Ragazzo TG, Paranagua-Vezozzo D, Lima FR, de Campos Mazo DF, Pessoa MG, Oliveira CP, Alves VAF, Carrilho FJ. Accuracy of transient elastography-FibroScan(R), acoustic radiation force impulse (ARFI) imaging, the enhanced liver fibrosis (ELF) test, APRI, and the FIB-4 index compared with liver biopsy in patients with chronic hepatitis C. Clinics (Sao Paulo). 2017;72(9):516-25.

12. $\mathrm{Yu} \mathrm{JH}$, Lee Jl. Current role of transient elastography in the management of chronic hepatitis B patients. Ultrasonography. 2017;36(2):86-94.

13. Wang J, Xia J, Yan $X$, et al. Plateletcrit as a potential index for predicting liver fibrosis in chronic hepatitis B. J Viral Hepat. 2020;27(6):602-9.

14. Kattan MW, Scardino PT. Evidence for the usefulness of nomograms. Nat ClinPractUrol. 2007:4(12):638-9.

15. Huang JL, Fu YP, Jing CY, Yi Y, Sun J, Gan W, Lu ZF, Zhou J, Fan J, Qiu SJ. A novel and validated prognostic nomogram based on liver fibrosis and tumor burden for patients with hepatocellular carcinoma after curative resection. J SurgOncol. 2018;117(4):625-33.

16. Huang YQ, Liang CH, He L, Tian J, Liang CS, Chen X, Ma ZL, Liu ZY. Development and validation of a radiomicsnomogram for preoperative prediction of lymph node metastasis in colorectal cancer. J ClinOncol. 2016;34(18):2157-64

17. Kattan MW, Sternberg CN, Mehmud F, Bhatt K, McCann L, Motzer RJ. Development and validation of a prognostic nomogram for progression-free survival in patients with advanced renal cell carcinoma treated with pazopanib. Oncology. 2015;89(4):235-41.

18. Kent MS, Mandrekar SJ, Landreneau R, et al. A nomogram to predict recurrence and survival of high-risk patients undergoing sublobar resection for lung cancer: an analysis of a multicenter prospective study (ACOSOG Z4032). Ann ThoracSurg. 2016;102(1):239-46.

19. Wang Y, Li J, Xia Y, et al. Prognostic nomogram for intrahepatic cholangiocarcinoma after partial hepatectomy. J ClinOncol. 2013;31(9):1188-95.
20. Ding RR, Zheng JM, Huang D, et al. INR-to-platelet ratio (INPR) as a novel noninvasive index for predicting liver fibrosi in chronic hepatitis B. Int J Med Sci. 2021;18(5):1159-66.

21. Scheuer PJ. Classification of chronic viral hepatitis: a need for reassessment. J Hepatol. 1991;13(3):372-4.

22. Boursier J, de Ledinghen $V$, Leroy $V$, et al. A stepwise algorithm using an at-a-glance first-line test for the non-invasive diagnosis of advanced liver fibrosis and cirrhosis. J Hepatol. 2017;66(6):1158-65.

23. Sterling RK, Lissen E, Clumeck N, et al. Development of a simple noninvasive index to predict significant fibrosis in patients with HIV/HCV coinfection. Hepatology. 2006;43(6):1317-25.

24. Cross TJ, Rizzi P, Berry PA, Bruce M, Portmann B, Harrison PM. King's Score: an accurate marker of cirrhosis in chronic hepatitis $C$. Eur J GastroenterolHepatol. 2009;21(7):730-8.

25. Takaki S, Kawakami Y, Miyaki D, et al. Non-invasive liver fibrosis score calculated by combination of virtual touch tissue quantification and serum liver functional tests in chronic hepatitis C patients. Hepatol Res. 2014:44(3):280-7

26. Baranova A, Lal P, Birerdinc A, et al. Non-Invasive markers for hepatic fibrosis. BMC Gastroenterol. 2011;11:91-6.

27. Cross TJ, Calvaruso V, Maimone S, et al. Prospective comparison of Fibroscan, King's score and liver biopsy for the assessment of cirrhosis in chronic hepatitis C infection. J Viral Hepat. 2010;17(8):546-54.

28. Sugimoto M, Saiki H, Tamai A, et al. Ventricular fibrogenesis activity assessed by serum levels of procollagen type III N-terminal amino peptide during the staged Fontan procedure. J ThoracCardiovascSurg. 2016:151(6):1518-26.

29. Tanwar S, Trembling PM, Guha IN, et al. Validation of terminal peptide of procollagen III for the detection and assessment of nonalcoholicsteatohepatitis in patients with nonalcoholic fatty liver disease. Hepatology. 2013;57(1):103-11.

30. Camacho VR, Silveira TR, Oliveira JR, Barros SG, Cerski CT. Relationship between serum concetrations of type III procollagen, hyluronic acid and histopathological findings in the liver of HCV-positive blood donors. ArqGastroenterol. 2007:44(2):118-22.

31. Pares A, Deulofeu R, Gimenez A, Caballeria L, Bruguera M, Caballeria J, Ballesta AM, Rodes J. Serum hyaluronate reflects hepatic fibrogenesis in alcoholic liver disease and is useful as a marker of fibrosis. Hepatology. 1996;24(6):1399-403.

32. Remmel T, Remmel H, Salupere V. Aminoterminalpropeptide of type III procollagen and hyaluronan in patients with primary biliary cirrhosis: markers of fibrosis in primary biliary cirrhosis. J GastroenterolHepatol. 1996;11(11):1016-20

33. Trepo E, Potthoff $A$, Pradat $P$, et al. Role of a cirrhosis risk score for the early prediction of fibrosis progression in hepatitis $C$ patients with minimal liver disease. J Hepatol. 2011;55(1):38-44.

34. Parkes J, Guha IN, Roderick P, et al. Enhanced Liver Fibrosis (ELF) test accurately identifies liver fibrosis in patients with chronic hepatitis C. J Viral Hepat. 2011;18(1):23-31.

35. Nielsen MJ, Veidal SS, Karsdal MA, et al. Plasma Pro-C3 (N-terminal type III collagen propeptide) predicts fibrosis progression in patients with chronic hepatitis C. Liver Int. 2015;35(2):429-37.

36. Daniels SJ, Leeming DJ, Eslam M, et al. ADAPT: an algorithm incorporating PRO-C3 accurately identifies patients with NAFLD and advanced fibrosis. Hepatology. 2019;69(3):1075-86.

37. Teshale E, Lu M, Rupp LB, et al. APRI and FIB-4 are good predictors of the stage of liver fibrosis in chronic hepatitis B: the Chronic Hepatitis Cohort Study (CHeCS). J Viral Hepat. 2014;21(12):917-20.

38. Shaheen AA, Myers RP. Diagnostic accuracy of the aspartate aminotransferase-to-platelet ratio index for the prediction of hepatitis C-related fibrosis: a systematic review. Hepatology. 2007;46(3):912-21.

39. Xiao G, Yang J, Yan L. Comparison of diagnostic accuracy of aspartate aminotransferase to platelet ratio index and fibrosis-4 index for detecting liver fibrosis in adult patients with chronic hepatitis $B$ virus infection: a systemic review and meta-analysis. Hepatology. 2015:61(1):292-302.

40. Polyzos SA, Slavakis A, Koumerkeridis G, Katsinelos P, Kountouras J. Noninvasive liver fibrosis tests in patients with nonalcoholic fatty liver disease: an external validation cohort. HormMetab Res. 2019;51(2):134-40 
41. Zhou YJ, Ye FZ, Li YY, et al. Individualized risk prediction of significant fibrosis in non-alcoholic fatty liver disease using a novel nomogram. United European Gastroenterol J. 2019;7(8):1124-34.

42. Lemoine M, Shimakawa Y, Nayagam S, et al. The gamma-glutamyltranspeptidase to platelet ratio (GPR) predicts significant liver fibrosis and cirrhosis in patients with chronic HBV infection in West Africa. Gut. 2016;65(8):1369-76.

43. Lemoine M, Thursz M, Mallet V, Shimakawa Y. Diagnostic accuracy of the gamma-glutamyltranspeptidase to platelet ratio (GPR) using transient elastography as a reference. Gut. 2017;66(1):195-6.
44. Cai YJ, Dong JJ, Wang XD, et al. A diagnostic algorithm for assessment of liver fibrosis by liver stiffness measurement in patients with chronic hepatitis B. J Viral Hepat. 2017;24(11):1005-15.

\section{Publisher's Note}

Springer Nature remains neutral with regard to jurisdictional claims in published maps and institutional affiliations.
Ready to submit your research? Choose BMC and benefit from:

- fast, convenient online submission

- thorough peer review by experienced researchers in your field

- rapid publication on acceptance

- support for research data, including large and complex data types

- gold Open Access which fosters wider collaboration and increased citations

- maximum visibility for your research: over $100 \mathrm{M}$ website views per year

At BMC, research is always in progress.

Learn more biomedcentral.com/submissions 